\title{
THE SIMPLE-ZERO THEOREM FOR SUPPORT POINTS IN $\Sigma$
}

\author{
Y. J. LEUNG AND G. SCHOBER \\ (Communicated by Irwin Kra)
}

\begin{abstract}
The purpose of this article is to confirm a simple-zero conjecture raised by the second author for support points in $\Sigma$. The conjecture implies that the omitted arcs of an extremal function in $\Sigma$ for a linear problem can branch out in, at most, three equiangular directions at a time.
\end{abstract}

\section{INTRODUCTION}

Let $\Sigma$ be the class of functions $f(z)=z+b_{0}+b_{1} / z+\cdots$ analytic and univalent in $\Delta=\{z:|z|>1\}$. The Koebe function $K(z)=z+2+1 / z$, which omits the line segment $[0,4]$ from its range, is a prominent member of $\Sigma$. Let $L$ be a continuous linear functional defined on the space of functions analytic in $\Delta$, with the topology of locally uniform convergence. In addition, assume that $L$ is nonconstant on $\Sigma$ and that $L(1)=0$. A function $f$ in $\Sigma$ is called a support point of $\Sigma$ for $L$ if $\operatorname{Re} L(f) \geq \operatorname{Re} L(g)$ for all $g$ in $\Sigma$. It follows from Schiffer's boundary variation (cf. [7]) that the continuum $\Gamma$ omitted by a support point $f$ consists of analytic arcs lying on trajectories of the quadratic differential $L\left(1 /(f-w) d w^{2}\right.$.

As a function of $w, L(1 /(f-w))$ has an analytic extension to some open region containing the omitted set $\Gamma$. Thus the structure theory of quadratic differentials [9] shows that the analytic arcs of $\Gamma$ are joined to one another only at the zeros of $L(1 /(f-w))$. At a zero of order $k \geq 1$, at most $k+2$ arcs may join at a time in a subset of $k+2$ equiangular directions. For example, the coefficient functional $L(f)=b_{3}$ leads to a quadratic differential of the form $\left(w^{2}-b_{1}\right) d w^{2}$. The omitted set of a support point for this functional forks at each of the two simple zeros at $\pm \sqrt{b_{1}}$ in three equiangular directions. Further examples of support points can be found in $[3,4]$.

Based on various known examples, the second author of this article conjectured $[4,8]$ that the quadratic differential $L(1 /(f-w)) d w^{2}$ associated with a support point $f$ in $\Sigma$ can have only simple zeros on the set $\Gamma$ omitted by $f$.

Received by the editors December 7, 1987.

1980 Mathematics Subject Classification (1985 Revision). Primary 30C75.

This work was supported in part by grants from the National Science Foundation. 
It is the purpose of this article to verify this simple-zero conjecture. Our main result is:

Theorem 1. If $f$ is a support point in $\Sigma$ for some linear functional $L$, then its associated quadratic differential $L(1 /(f-w)) d w^{2}$ can have only simple zeros on the omitted set of $f$.

Geometrically, this theorem implies that the omitted arcs can fork at the simple zeros in, at most, three equiangular directions. Some supporting evidence had been presented recently in $[4,5]$. For example, it is known that if $\Gamma$ contains a zero of order $k \geq 2$ at $w_{0}$, then it cannot contain a subset of arcs branching out symmetrically at $w_{0}$. On the other hand, it should be pointed out that $L(1 /(f-w))$ may have zeros of any order off of $\Gamma$ [5, Theorem 5.1]. In addition, a result of Abu-Muhanna [1] shows that any single analytic slit of mapping radius one is a support point for some linear functional $L$.

The proof of Theorem 1 consists of two parts. First, we use a technique of Schiffer's [2, pp. 216-218] to convert the conjecture into a coefficient problem. Next, we present a form of the second variation of the Koebe function to solve this coefficient problem.

\section{SCHIFFER'S TRUNCATION VARIATION}

For completeness, we shall present Schiffer's truncation variation (cf. also $[4, \mathrm{pp} .57-58])$ in order to convert the simple-zero conjecture into a coefficient problem for functions analytic and univalent in the complement of the line segment $[0,4]$.

After a translation, we may assume that $w=0$ is omitted by a support point $f$ for a functional $L$ and is a zero of order $k$ for the function $L(1 /(f-w))$. Expanding $L(1 /(f-w))$ about $w=0$, we see that $L\left(f^{-j}\right)=0$ for $j=$ $0,1, \ldots, k$ and $L\left(f^{-k-1}\right) \neq 0$. Furthermore, after rotating the functional and the function, we may assume also that $L\left(f^{-k-1}\right)>0$ and that one of the omitted arcs emanates from the origin in the positive horizontal direction.

To apply Schiffer's truncation, we consider a disk $|w| \leq \delta$ for a sufficiently small positive $\delta$. We delete all the arcs of $\Gamma$ except for that connected part of the arc emanating from the origin in the positive horizontal direction that lies inside the disk. Designate this subarc by $\Gamma_{\delta}$, and let $F_{\delta}$ be the conformal map from $\Delta$ onto the complement of $\Gamma_{\delta}$ with an expansion of the form $F_{\delta}(z)=$ $\rho z+O(1)$ around infinity and $\rho>0$. Then $F_{\delta}$ and the original $f$ are related by a Schwarz function $\omega: \Delta \rightarrow \Delta$ satisfying $F_{\delta} \circ \omega=f$ and $\omega^{\prime}(\infty)=1 / \rho$. As $\delta \rightarrow 0$, we have $\rho \rightarrow 0$. The function $f_{\delta}=(1 / \rho) F_{\delta}$ is a support point in $\Sigma$ for the functional $L_{\delta}$ defined by $L_{\delta}(g)=L(\rho g \circ \omega)$. Let us designate the set omitted by $f_{\delta}$ to be $\gamma_{\delta}$. It is obtained from dilating $\Gamma_{\delta}$ by the factor $1 / \rho$. Since $\gamma_{\delta}$ is an analytic arc emanating from $w=0$ in the positive horizontal direction, it approaches the line segment $[0,4]$ as $\delta \rightarrow 0$. By the Carathéodory convergence theorem, $f_{\delta}$ approaches the Koebe function $K(z)=z+2+1 / z$ as $\delta \rightarrow 0$. 
Let $F$ be analytic and univalent in the complement of $\gamma_{\delta}$ and have an expansion of the form $F(w)=w+\sum_{j=0}^{\infty} C_{j}(\delta) w^{-j}$ around infinity. Then $f_{\delta}^{*}=F \circ f_{\delta}$ serves as a variation of $f_{\delta}$, and so $\operatorname{Re} L_{\delta}\left(f_{\delta}^{*}\right) \leq \operatorname{Re} L_{\delta}\left(f_{\delta}\right)$. Using the definition of $L_{\delta}$, we rewrite this inequality as $\operatorname{Re} L\left(f_{\delta}^{*} \circ \omega\right) \leq \operatorname{Re} L\left(f_{\delta} \circ\right.$ $\omega)$. Since $f_{\delta}^{*} \circ \omega=f_{\delta} \circ \omega+\sum_{j=0}^{\infty} C_{j}(\delta) \rho^{j} f^{-j}$ for $|f|>\delta$, we conclude that $\operatorname{Re}\left\{\sum_{j=0}^{\infty} C_{j}(\delta) \rho^{j} L\left(f^{-j}\right)\right\} \leq 0$ when $\delta$ is sufficiently small. From the assumption on the order of the zero at $w=0$, the terms of this series with index $j \leq k$ are zero. Therefore, we have

$$
\operatorname{Re}\left\{C_{k+1}(\delta)\right\} \rho^{k+1} L\left(f^{-k-1}\right)+O\left(\rho^{k+2}\right) \leq 0,
$$

where $L\left(f^{-k-1}\right)$ is positive. Dividing by $\rho^{k+1}$ and letting $\delta \rightarrow 0$ leads us to the conclusion that $\operatorname{Re} C_{k+1} \leq 0$ for any univalent function $F(w)=w+$ $\sum_{j=0}^{\infty} C_{j} w^{-j}$ in the complement of $\gamma_{0}=[0,4]$. Hence, the presence of a $k$ th order zero implies the following:

If the omitted set $\Gamma$ of a support point for some functional contains a kth order zero of its associated quadratic differential $L(1 /(f-w)) d w^{2}$, then the inequality

$$
\operatorname{Re} C_{k+1} \leq 0
$$

is valid for every function $F$ analytic and univalent in the complement of the line segment $[0,4]$ with expansion $F(w)=w+\sum_{j=0}^{\infty} C_{j} w^{-j}$ in a neighborhood of infinity.

In the next section, for each $k \geq 2$ we shall use a second-order variation of the identity to show that there are functions $F$ with $k+1$ st coefficient $C_{k+1}$ satisfying $\operatorname{Re} C_{k+1}>0$. Combined with the statement above, this eliminates the presence of all $k$ th order zeros of $L(1 /(f-w)) d w^{2}$ in the omitted set for $k \geq 2$.

\section{SECOND-ORDER VARIATION}

To derive variational formulas for mappings defined in the complement of the segment $[0,4]$, we shall use the Loewner equation for the familiar class $S$ of univalent analytic functions $f$ defined in the unit disk $\mathbf{D}=\{z:|z|<1\}$, with $f(0)=0$ and $f^{\prime}(0)=1$. For simplicity, let $\kappa=\kappa(t)$ be a continuous complex-valued function on $[0, \infty)$ with $|\kappa(t)|=1$. Then the differential equation

$$
\frac{\partial f}{\partial t}=z\left(\frac{1+\kappa z}{1-\kappa z}\right) \frac{\partial f}{\partial z}, \quad t \geq 0, z \in \mathbf{D},
$$

has a solution $f(z, t)$ such that the functions $e^{-t} f(\cdot, t)$ belongs to $S$ (cf. [6, $\S 6.1])$. In particular, $f(\cdot, 0)$ belongs to $S$.

In order to refer the Loewner chain $f(\cdot, t)$ to the complement of the segment $[0,4]$, we shall use the function $K(z)=z+2+1 / z$, which also maps $D \backslash\{0\}$ onto the complement of $[0,4]$. Denote the inverse of this mapping by $S$. 
Then we have $S(w)=\frac{1}{2}(w-2-w \sqrt{1-4 / w})$, where the branch of the square root is chosen so that $\sqrt{1}=1$. The corresponding Loewner chain of mappings $F(\cdot, t)$ in the complement of $[0,4]$ are defined by $F(w, t)=1 / f(S(w), t)$. From (2) and the chain rule one finds that they satisfy the differential equation

$$
\frac{\partial F}{\partial t}=\left(S(w)-\frac{1}{S(w)}\right)\left(\frac{1+\kappa S(w)}{1-\kappa S(w)}\right) \frac{\partial F}{\partial w}, \quad t \geq 0,
$$

and $e^{t} F(w, t)=w+O(1)$ as $w \rightarrow \infty$. In particular, $F(\cdot, 0)$ is a competitor for the inequality (1).

We choose $\kappa(t)=e^{i \varepsilon \theta(t)}$, with $\varepsilon$ being a small real parameter and $\theta$ a bounded continuous function of $t$. Then we have the expansions $\kappa=1+i \varepsilon \theta-$ $\frac{1}{2} \varepsilon^{2} \theta^{2}+O\left(\varepsilon^{3}\right)$ and

$$
\frac{1+\kappa S}{1-\kappa S}=\frac{1+S}{1-S}+i \varepsilon \theta \frac{2 S}{(1-S)^{2}}-\varepsilon^{2} \theta^{2} \frac{S(1+S)}{(1-S)^{3}}+O\left(\varepsilon^{3}\right)
$$

as $\varepsilon \rightarrow 0$. In this case, the differential equation (3) is of the form

$$
\frac{\partial F}{\partial t}=\left[-\frac{(1+S)^{2}}{S}-2 i \varepsilon \theta \frac{1+S}{1-S}+\varepsilon^{2} \theta^{2}\left(\frac{1+S}{1-S}\right)^{2}+O\left(\varepsilon^{3}\right)\right] \frac{\partial F}{\partial w}
$$

for the function

$$
F(w, t, \varepsilon)=\phi(w, t)+\varepsilon V(w, t)+\varepsilon^{2} Q(w, t)+O\left(\varepsilon^{3}\right) .
$$

Since $(1+S)^{2} / S=w$, the differential equation (4) for $\varepsilon=0$ becomes $\partial \phi / \partial t=$ $-w \partial \phi / \partial w$. It follows that $e^{t} \phi(w, t)=w$, and so the functions $e^{t} F(\cdot, t, \varepsilon)$ are normalized variations of the identity mapping.

A comparison of the coefficients of $\varepsilon$ and $\varepsilon^{2}$ in (4) leads to the differential equations

$$
\begin{aligned}
& \frac{\partial V}{\partial t}=-w \frac{\partial V}{\partial w}-2 i e^{-t} \theta \frac{1+S}{1-S}, \\
& \frac{\partial Q}{\partial t}=-w \frac{\partial Q}{\partial w}-2 i \theta \frac{1+S}{1-S} \frac{\partial V}{\partial w}+e^{-t} \theta^{2}\left(\frac{1+S}{1-S}\right)^{2},
\end{aligned}
$$

for the first and second variations. By expressing $S$ explicitly in terms of $w$, these equations can be rewritten as

$$
\begin{aligned}
& \frac{\partial V}{\partial t}=-w \frac{\partial V}{\partial w}-2 i e^{-t} \theta \frac{1}{\sqrt{1-4 / w}}, \\
& \frac{\partial Q}{\partial t}=-w \frac{\partial Q}{\partial w}-2 i \theta \frac{1}{\sqrt{1-4 / w}} \frac{\partial V}{\partial w}+e^{-t} \theta^{2} \frac{1}{1-4 / w} .
\end{aligned}
$$

Since $F(w, t, \varepsilon)-\phi(w, t)=O(1)$ as $w \rightarrow \infty$, the expansions of $V$ and $Q$ around infinity are of the form $V(w, t)=\sum_{n=0}^{\infty} V_{n}(t) w^{-n}$ and $Q(w, t)=$ $\sum_{n=0}^{\infty} Q_{n}(t) w^{-n}$. It follows from the system of differential equations (5) that

$$
V_{n}^{\prime}=n V_{n}-2 i e^{-t} \theta d_{n}
$$


and

$$
Q_{n}^{\prime}=n Q_{n}+2 i \theta \sum_{j=1}^{n-1} j d_{n-1-j} V_{j}+4^{n} e^{-t} \theta^{2}
$$

where

$$
\frac{1}{\sqrt{1-4 / w}}=\sum_{n=0}^{\infty} d_{n} w^{-n} \quad \text { with } \quad d_{n}=\frac{(2 n) !}{(n !)^{2}} .
$$

If $w$ is restricted, say, to a large circle, then $e^{t} F(w, t, \varepsilon)$ is uniformly bounded. Therefore the coefficients $V_{n}(t)$ and $Q_{n}(t)$ vanish at $t=\infty$. Thus this system can be integrated to yield

$$
V_{n}(0)=2 i d_{n} \int_{0}^{\infty} e^{-(n+1) t} \theta(t) d t
$$

and

$$
\begin{aligned}
Q_{n}(0) & =-4^{n} \int_{0}^{\infty} e^{-(n+1) t} \theta^{2}(t) d t \\
& +4 \sum_{j=1}^{n-1} j d_{j} d_{n-1-j} \int_{0}^{\infty} \int_{t}^{\infty} e^{-(n-j) t-(j+1) s} \theta(t) \theta(s) d s d t
\end{aligned}
$$

In the expansion of $F(w, 0, \varepsilon)=w+\sum_{n=0}^{\infty} C_{n}(0, \varepsilon) w^{-n}$, the coefficients are of the form $C_{n}(0, \varepsilon)=\varepsilon V_{n}(0)+\varepsilon^{2} Q_{n}(0)+O\left(\varepsilon^{3}\right)$. Evidently, $V_{n}(0)$ is purely imaginary and $Q_{n}(0)$ is real, so $\operatorname{Re} C_{n}(0, \varepsilon)=\varepsilon^{2} Q_{n}(0)+O\left(\varepsilon^{3}\right)$. For each $n \geq 3$, we shall find a function $\theta$ such that $Q_{n}(0)>0$. This will show the existence of a function $F(\cdot, 0, \varepsilon)$, nearby the identity function, with $\operatorname{Re} C_{n}(0, \varepsilon)$ positive. As pointed out at the end of $\S$ II, this will complete the proof of Theorem 1.

Substitute $\theta(t)=e^{(2-\delta) t}$ into (6), and discard from the sum all terms except the one for which $j=1$. Then we have

$$
\begin{aligned}
Q_{n}(0) & \geq-4^{n} \int_{0}^{\infty} e^{-(n-3+2 \delta) t} d t+4 d_{1} d_{n-2} \int_{0}^{\infty} \int_{t}^{\infty} e^{-(n-3+\delta) t-\delta s} d s d t \\
& =\frac{1}{n-3+2 \delta}\left[-4^{n}+\frac{4 d_{1} d_{n-2}}{\delta}\right]
\end{aligned}
$$

for $n>3-2 \delta$, where $d_{1}$ and $d_{n-2}$ are positive. When $n \geq 3$ and $\delta$ is positive and sufficiently small, it follows that $Q_{n}(0)>0$. Although this is the desired conclusion, the function $\theta$ that was chosen is not bounded. However, the truncation $\theta_{N}(t)=\min \{N, \theta(t)\}$ is bounded, and an application of the dominated convergence theorem leads to the same conclusion that $Q_{n}(0)$ is positive for some $\theta_{N}$.

Thus for each $n \geq 3$, we have shown the existence of a function $F$, analytic and univalent in the complement of $[0,4]$, such that the coefficient $C_{n}$ in the expansion $F(w)=w+\sum_{j=0}^{\infty} C_{j} w^{-j}$ around $w=\infty$ satisfies $\operatorname{Re} C_{n}>0$. Therefore we have eliminated the presence of $n-1$ st order zeros for each $n \geq 3$. This completes the proof of Theorem 1 . 
Remark. The search for a positive $Q_{n}(0)$ can be rephrased as an eigenvalue problem for an integral equation. After a change of variables, one can ask for the maximum of

$$
\frac{-4^{n} \int_{0}^{1} y^{n} \vartheta^{2}(y) d y+4 \sum_{j=1}^{n-1} j d_{j} d_{n-1-j} \int_{0}^{1} \int_{0}^{y} x^{j} y^{n-1-j} \vartheta(x) \vartheta(y) d x d y}{\int_{0}^{1} \vartheta^{2}(y) d y}
$$

for functions $\vartheta$, say, in $\mathscr{L}^{2}[0,1]$. If $\lambda$ denotes this maximum, then the Euler equation for this problem is the integral equation

$$
\begin{array}{r}
-4^{n} y^{n} \vartheta(y)+2 \sum_{j=1}^{n-1} j d_{j} d_{n-1-j}\left\{y^{n-1-j} \int_{0}^{y} x^{j} \vartheta(x) d x+y^{j} \int_{y}^{1} x^{n-1-j} \vartheta(x) d x\right\} \\
=\lambda \vartheta(y) .
\end{array}
$$

The range of the function $-4^{n} y^{n}$ and, hence, the continuous spectrum of the integral equation is the interval $\left[-4^{n}, 0\right]$. Implicit in our work is the existence of a positive eigenvalue.

\section{REFERENCES}

1. Y. Abu-Muhanna and Y. J. Leung, On analytic slit mappings in the class $\Sigma$, Proc. Amer. Math. Soc. 99 (1987), 44-48.

2. A. Chang, M. M. Schiffer and G. Schober, On the second variation for univalent functions, J. Analyse Math. 40 (1981), 203-238.

3. W. E. Kirwan and G. Schober, New inequalities from old ones, Math. Z. 180 (1982), 19-40.

4. Y. J. Leung and G. Schober, Low order coefficient estimates in the class $\Sigma$, Ann. Acad. Sci. Fenn. Ser. AI Math. 11 (1986), 36-61.

5. _ On the structure of support points in the class $\Sigma$, J. Analyse Math. 46 (1986), 176-193.

6. Chr. Pommerenke, Univalent functions, Vandenhoeck \& Ruprecht, Göttingen, 1975.

7. G. Schober, Univalent functions-Selected topics, Lecture notes in math. \#478, Springer-Verlag, Berlin, 1975.

8. _ Some conjectures for the class $\Sigma$, in Topics in complex analysis, Contemporary Math. 38 (1985), 13-21.

9. K. Strebel, Quadratic differentials, Springer-Verlag, Berlin, 1984.

University of Delaware, Newark, Delaware 19716

INDIANA UNIVERSITY, BLOOMINGTON, INDIANA 47405 